\title{
Non-contact monitoring for assessing potential bridge damages
}

\author{
Boštjan Kovačič ${ }^{1, *}$, Luka Muršec ${ }^{1}$, Sebastian Toplak ${ }^{1}$ and Samo Lubej ${ }^{1}$ \\ ${ }^{1}$ University of Maribor, Faculty of Civil Engineering, Transportation Engineering and Architecture, \\ 17, Smetanova, 2000, Maribor, Slovenia
}

\begin{abstract}
Structural monitoring of objects is primarily executed to assess external and internal effects on the object, in order to ensure the safety of people, animals, and material assets. Such monitoring can be executed through various methods, depending on the object, conditions for execution, and purpose of the monitoring. In this case, the focus is on the execution of the monitoring of Maribor footbridge, where the dynamic effects of the object are monitored. For this purpose, geophone, accelerometer, and geodetic methods - using Global Navigation Satellite System (GNSS) and Robotic Total Station (RTS) equipment - are used, of which one is controlled by the additional programme GeoComZG. The emphasis of our experiment is on the application of non-contact geodetic methods, with which the measurements of dynamic response are typically performed, as they enable measurements up to 30 and $100 \mathrm{~Hz}$ with RTS and GNSS, respectively. In this article, the application of various procedures of non-contact data capture on the footbridge are detailed and a comparison and analysis of the obtained values for monitoring the dynamic response of the structure are presented.
\end{abstract}

\section{Introduction}

Modern monitoring techniques for bridges are typically based on the measurement of the displacements and vibrations. Data on the static and dynamic conduct of the structure under load are usually obtained from burdening tests. With the obtained data from the burdening tests and continued monitoring, data on the long-term conduct of the objects can be obtained. This is very important for highly loaded objects, such as those on highways or railways. The technological development of measuring equipment has intervened in the area of geodetic instruments; until a few years ago, it was only possible the determine the vertical and horizontal displacements and vibrations in the range of their natural frequencies. With the development of the interferometric radar, and the consequent increased resolution, faster scanning, and automatic prism tracking, very accurate static measurements and measurements of the dynamic response of structures at various monitoring points can be performed $[1,2]$. It is necessary to obtain high-quality data about the conduct of the structure on parts which, with other methods, is practically impossible

* Corresponding author: bostjan.kovacic@um.si 
due to the inaccessibility or the complexity of the structure; however, they can be obtained with geodetic methods in a very simple way.

Geodetic monitoring with GNSS of the dynamic response of structures was, at first, directed only at flexible building structures, such as suspension bridges, skyscrapers, or towers [3-7]. These structures are characterized by larger displacements and lower oscillation frequencies. Modern GNSS technology enables us to capture data with frequencies up to $100 \mathrm{~Hz}$ [8].

A recent alternative geodetic method is the application of robotic total stations (RTS) or robotic tachometers. This method was successfully used for numerous measurements of the semi-static $[9,10]$ and dynamic responses in the size class of a few centimeters $[11,12]$. A more difficult task for robotic total stations is the measurement of the dynamic parameters of shorter and stiffer bridging objects, which are characterized by higher natural and oscillation frequencies, as well as lower amplitudes. Current systematic experiments with RTS and the built-in hardware GeoCom have proven their ability to measure oscillations up to $20 \mathrm{~Hz}$ [13] and amplitudes of a few millimeters. Despite this, only a few experiments have been executed with a recording frequency of 5-7 Hz [14]. This fact appeals to the necessity of further analysis of the ability and accuracy of the instruments in measuring the dynamics of the building objects. The flaw of these methods is in their installation on difficult structures (for accelerometers and GNSS antennas) or special prisms (for RTS).

The robotic tachometer or robotic total station (RTS) is a new generation of geodetic instrument, which automatically captures data on the change of the length, horizontal angle, and vertical angle.

In the works $[15,16,17]$, the methods and results of studies into the possibility of application of Robotic Total Stations in monitoring prisms in motion have been described.

The authors of these works described mainly the non-synchronization error of the measuring instrument sensors, used for measuring lengths and directions, which led to a system error in determining the co-ordinates of the prism's position. In the works $[18,19]$, robotic total stations produced by various manufacturers for determining prism positions were tested, where the prism moved on right arcs and in certain directions at various velocities. In [20], the capabilities of an RTS in measuring high-frequency displacements in a horizontal direction was tested.

As for the biggest advantage of the RTS, it is necessary to emphasize their high measurement precision. For kinematic measurements with the purpose of making precise 3D measurements with the result displayed in real-time, RTS is considered to be the most convenient method. It is necessary to emphasize that many parameters affect the stated precision; depending on the RTS characteristics, the used prism, and atmospheric conditions during the measurement. The velocity of the prism's motion, the capability of RTS servomotors to monitor the prism in motion, the conduct of compensators during the movement, and sophistication of algorithms to monitor the prism in the case of signal break all influence the execution of kinematic measuring, as well.

The project of measuring the dynamic response of Wilford Bridge in Nottingham from 2002 [21] was among the first studies into the adequacy of RTS applications in measuring the dynamic responses of bridges. In the work, the possibilities and flaws of the application of RTS for these types of measurements were given.

The biggest flaw of the typical measuring instruments used is the slow and uneven execution of measurements. In [22], comparisons of the dynamic responses of a bridge, as determined with GPS, RTS, and accelerometers, are presented. The flaws of the GNSS method, such as uneven sampling after the removal of low-quality and defective measurements (due to, e.g., multi-path or bad satellite arrangement issues), the unreliability of the altitude component, and signal disturbances, were eliminated with the establishment 
of additional geodetic measurements using an RTS, which captured the position data in an automated manner.

This flaw has been eliminated with the development of technology and additional programs. If the structure, conditions, and (of course) time allow, it is the best to execute the measurements with various methods (GPS, RTS, and accelerometers) and compare the results, as has been shown in $[1,22]$. Recently, many authors [23-31] have dealt with the application and accuracy of the GNSS systems and therestic laser scanners in assessing the functions of various factors on objects in the field of monitoring.

In this article, the application of RTS (Leica instruments) and GNSS (Topcon system) in monitoring the dynamic conduct of footbridge object at Maribor will be shown. Physical methods, such as use of the Instantel, Mininimate Plus, and tri-axial Rotech Micro MON accelerometers for measuring the vibrations in all three axes were used to compare with and analyze the applicability of the geodetic results. The geodetic monitoring was executed with two instruments: a Leica TS50 and Leica TS30; with the TS50, an additional program/protocol GeoCOM/ZG, which enables up to 30 measurements per second, was used. The instrument was controlled by a computer using the GeoCOM protocol. The connection to the computer was established with a serial cable, and a baud rate setting of 115200 was used.

The program was developed at the Faculty of Geodesy in Zagreb and enabled measurement of the length, $\mathrm{Hz}$, and $\mathrm{V}$ of the angle per millisecond, or to measure only the $\mathrm{Hz}$ and $\mathrm{V}$ of the angle; where, due to the exclusion of the distance measurement, even more regular measurements per second can be performed. The second instrument, the Leica TS30, used the standard protocol GeoCom for the measurement, performing six measurements per second. The measuring point was the Leica GPR121 target. Both instruments used an autotarget tracking function. At the same position, a GNSS Topcon Hyper Pro system RTK with a scan speed of 20 reads per second was used, with both accelerometers activated.

From the mentioned works, research, and results, it can be concluded that the RTS method can be used as an autonomous and sufficiently accurate method for monitoring the regular dynamic responses of building structures, which is proven through the executed experiment.

\section{Materials and Methods for the Limitation of Dynamic Monitoring with RTS}

In the experiment where the dynamic response of the bridge was monitored to determine potential damages, we wanted to assess the potential influences of the prism on the course of the measurements. Thus, two various geodetic prisms were used. As, during the whole testing period and further execution of actual monitoring, we wanted to include the effect of the sampling frequency of the measuring machine on the obtained results, the tests were performed at both $6 \mathrm{~Hz}$ and $26 \mathrm{~Hz}$ data capture rates. Based on the performed testing it was concluded that, to execute practical monitoring of the actual structure, the difference in obtained results was insignificantly small and exceeded the declared accuracy of all relevant geodetic machine measurements.

For that purpose, a Leica TS50 with the GeoCOM/ZG protocol was tested, where each type of target measurement was performed for 5 minutes. The measurements were performed in two ways: with $6 \mathrm{~Hz}$ and $26 \mathrm{~Hz}$ sampling. Leica GPR121 and Optima prisms were used, as shown in Figure 1. In the article, the results with the Leica prism GPR121 will be presented, as it was used in the monitoring experiment. 


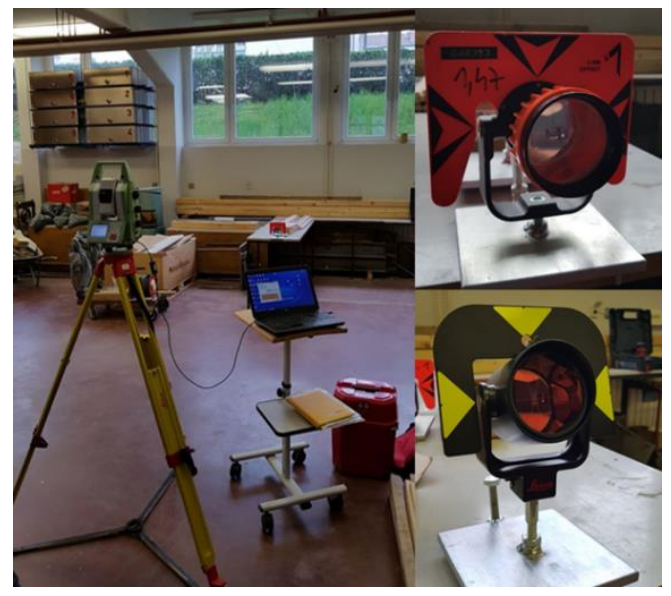

Fig. 1. The execution of the experiment in a laboratory (upper right: Optima; lower right: Leica GPR121).

In Figure 2 and Table 1, the results of the measurement with the RTS TS50 at $6 \mathrm{~Hz}$ and $26 \mathrm{~Hz}$ maximum measuring frequencies and at a distance of $5 \mathrm{~m}$ are shown. In the Figure, the measured vertical angles and coordinates of the point are shown in the horizontal plane of the timeline. Minimum and maximum values of certain co-ordinates are shown. In Figure 2, only a part of measurements are shown, with which the natural oscillation was assessed.
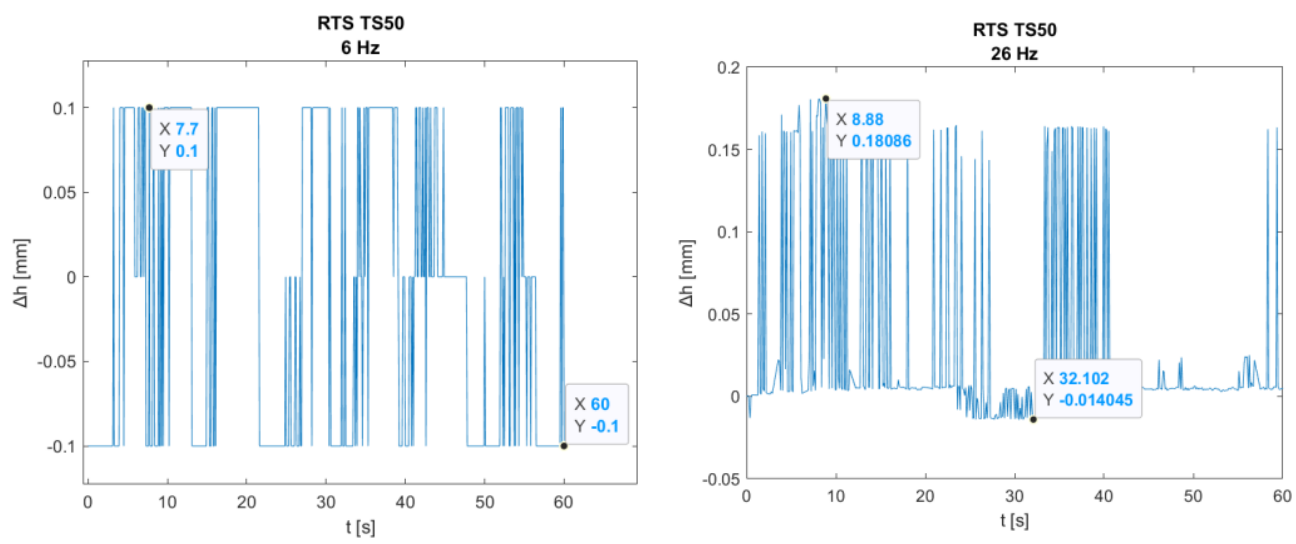

Fig. 2. Part of results of vertical deviation in assessing noise oscillation of the instrument and prisms at various frequencies.

Table 1. The results of noise oscillation of the instrument and prism, at various frequencies, for the Target Leica GPR121.

\begin{tabular}{|c|c|c|c|c|c|}
\hline \multirow{2}{*}{ Method } & \multirow{2}{*}{ Frequency } & \multicolumn{2}{|c|}{ Vertical displacement [mm] } & \multirow{2}{*}{$\begin{array}{c}\text { Standard deviation } \\
\text { [mm] }\end{array}$} \\
\cline { 3 - 5 } & & Min & Max & $\boldsymbol{\Delta}$ & \\
\hline RTS TS50 & $6 \mathrm{~Hz}$ & -0.1000 & 0.1000 & 0.2000 & 0.091517 \\
\hline GeoCOM/ZG & $26 \mathrm{~Hz}$ & -0.0141 & 0.1809 & 0.1950 & 0.055159 \\
\hline
\end{tabular}

Based on our test, it was concluded that, with the RTS method, the measurements with minimum frequency were performed $6 \mathrm{~Hz}$. In $[1,15,32,33]$, it was shown that, by 
considering the Nyquist theorem, only natural frequencies of less than $4,5 \mathrm{~Hz}$ can be detected using such a measurement rate [34].

\subsection{Automatic monitoring with the application of a non-contact system RTS and GNSS for assessing the damages on dynamic response}

Experimental measurements for the needs of monitoring were performed on the Maribor Footbridge. The Studenci Footbridge over the Drava River is a spatial stick bridging structure, designed for cyclists and pedestrians. The footbridge was built on this location in 1885 and has since been reconstructed many times into various static systems. It was built into its existing state in 2007 and, in 2008, its design was awarded a Footbridge Award as the Best New Pedestrian Bridge; it is shown in Figure 3.

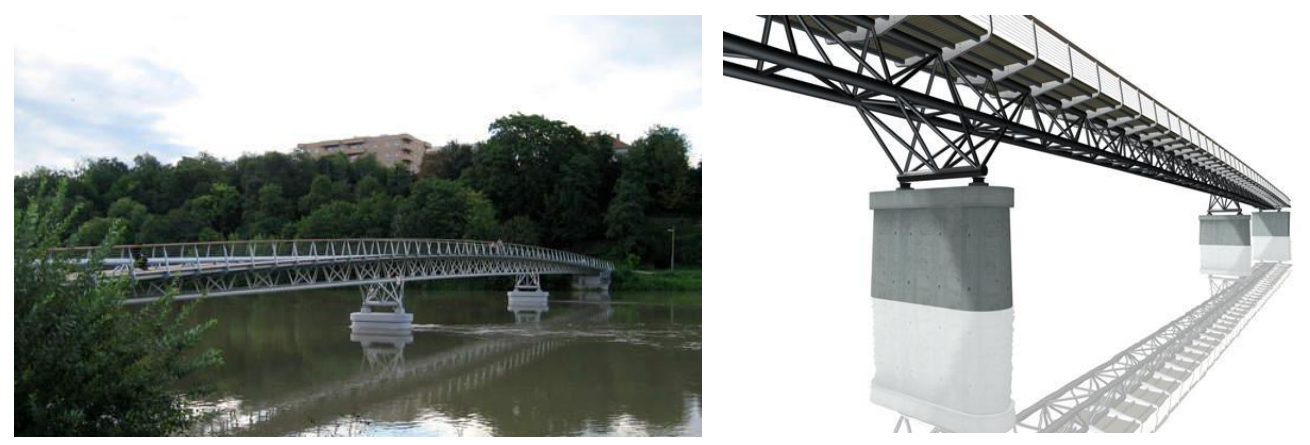

Fig. 3. Studenci Footbridge (Source: https://www.google.si).

The main structure is a spatial steel truss, which contains three longitudinal pipes: one pipe for the upper zone and two pipes for the lower zone, which relate to intermediate diagonals and bars.

The truss continues un-interruptedly over the existing supports with ranges in the structural axis of $42+42+42=126 \mathrm{~m}$. The upper walkway structure, with a width between from $3,2-5,8 \mathrm{~m}$, has a constant vertical curvature of $\mathrm{R}=4000 \mathrm{~m}$.

The measurements were performed on the first third of the range from the placement of the instruments. The biggest vibration and shift values were expected there. Vertical components, from which the dominant frequencies of the vibrations were calculated, were measured.

For monitoring of the dynamic response, more experiments were conducted, in which the oscillation was forced. A test was carried out, in which two people (of common weight $180 \mathrm{~kg}$ ) walked or ran in both directions. For the execution of this experiment, the geodetic instruments RTS Leica TS50 and TS30 and GNSS Topcon Hyper PRO were used. For comparison and analysis of the applicability of geodetic results, the Instantel and Rotech Micro Mon accelerometers were used for measuring the vibration in all three axes. The setup is shown in Figure 4. 


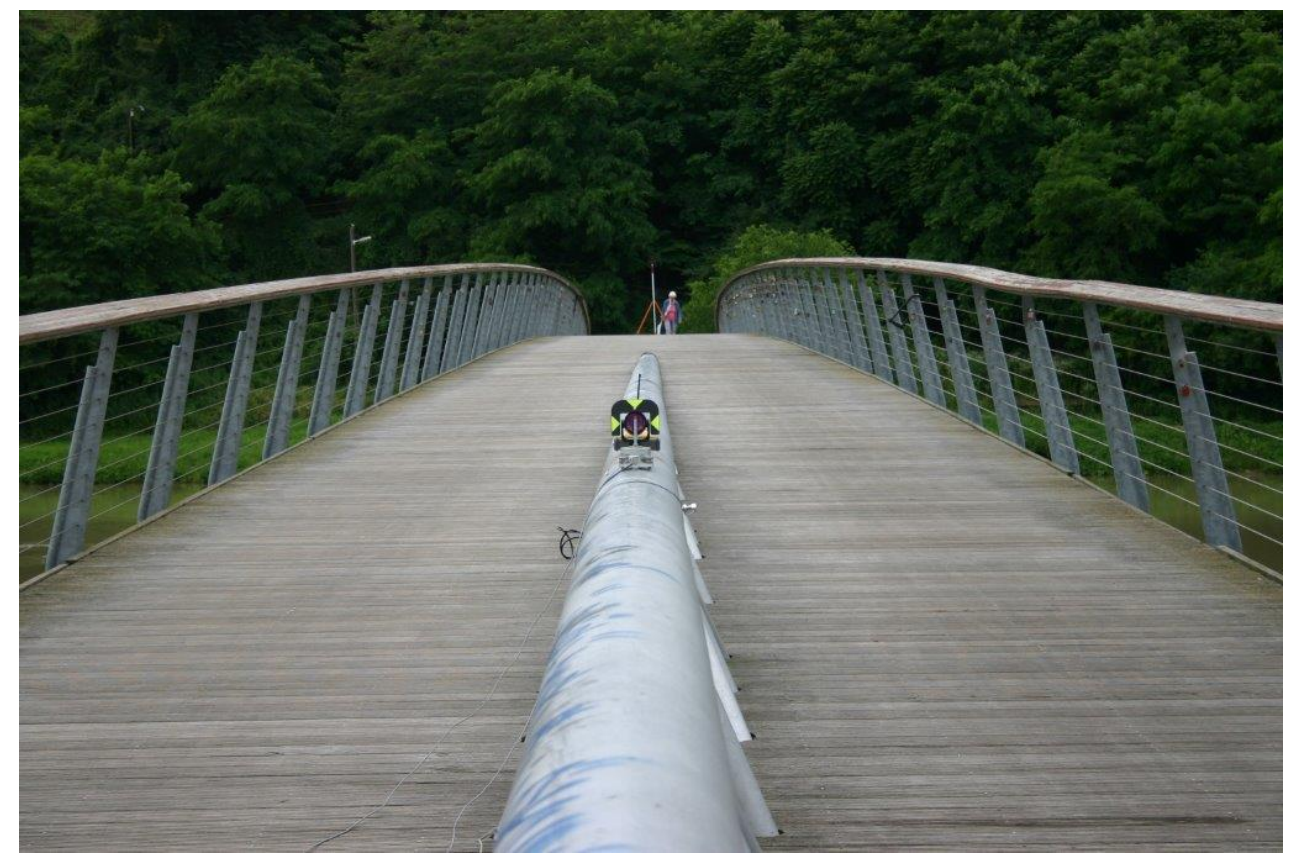

Fig. 4. The measuring equipment used (on the first third of the range from the measuring location).

Table 2 shows the used sensors and their measuring frequencies.

Table 2. Used sensors and their measuring frequencies.

\begin{tabular}{|c|l|c|}
\hline Sensors & \multicolumn{1}{|c|}{ Name } & Frequency [Hz] \\
\hline RTS50 & Leica TS50+ GeoCOM/ZG & 26 \\
\hline RTS30 & Leica TS30 & 20 \\
\hline GNSS & Topcon Hyper Pro & 250 \\
\hline ACC1 & Instantel & 2000 \\
\hline ACC2 & Rotech Micro Mon & \\
\hline
\end{tabular}

The basic instruments used for geodetic measuring of dynamic response in the experimental case were the robotic total stations Leica TS50 and TS30, which are shown in Figure 5. 

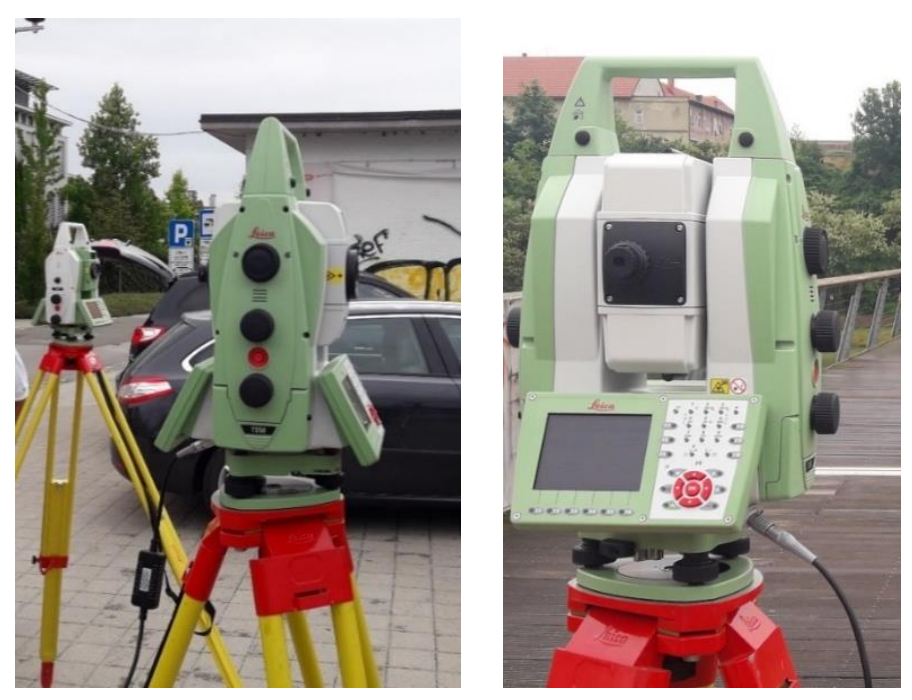

Fig. 5. Geodetic instruments Leica TS30/50 for monitoring.

Both instruments were placed on the non-deformable part, 19,5m away from the target. They were both following the same target Leica GPR121. These instruments were part of a new generation of robotic electronic instruments, which have the key characteristics of automatic target following and capability to sample spatial information (i.e., distances and angles) with a frequency of up to $20 \mathrm{~Hz}$. The instruments TS50 and TS30 have declared factory accuracies: according to ISO $17123-3$, for measuring angles $0.5 \mathrm{~s}$ or 0,15 mgons and, according to ISO $17123-4$, for distances $0,6 \mathrm{~mm}+1 \mathrm{ppm}$. TS30 followed the target at $6 \mathrm{~Hz}$, while the additional program GeoCom/Zg was used for TS50 which followed the target at $26 \mathrm{~Hz}$.

The GeoCom/Zg program for monitoring the targets in the motion with a read speed of per millisecond was developed at the University of Zagreb in Croatia at the Faculty of Geodesy. The program is connected to the instrument through the GEV269 cable. The instrument is controlled by the GeoCOM/ZG program, which has the options of reading length, angle, and time, or only angle (Figure 6).

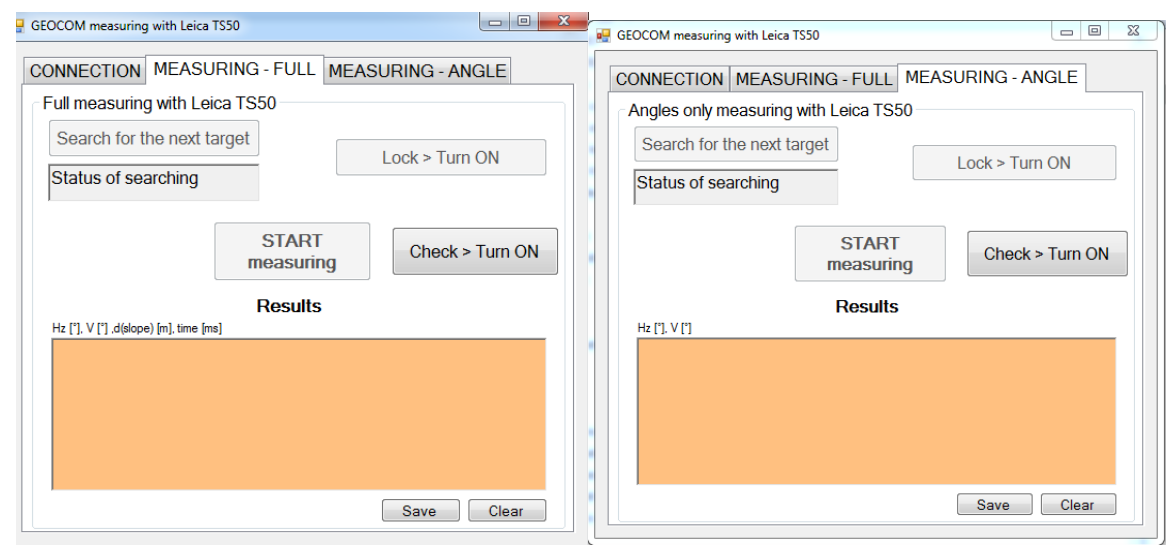

Fig. 6. The GeoCOM/ZG program with which the measurements were performed (left: all measurements, right: only measuring angles).

When testing the program, it was concluded that, by turning off the distance measurement, better readings were obtained. In determining the parameters of the dynamic 
response under equal burdening and oscillation, it was enough to capture the change of only the vertical angle over time.

The GNSS system is a known system. The RTK method was used, where the base station was set on the non-deformable field and the rover was attached to the structure (Figure 7). At the time of the experiment, the read speed was set to $0.1 \mathrm{~s}$.

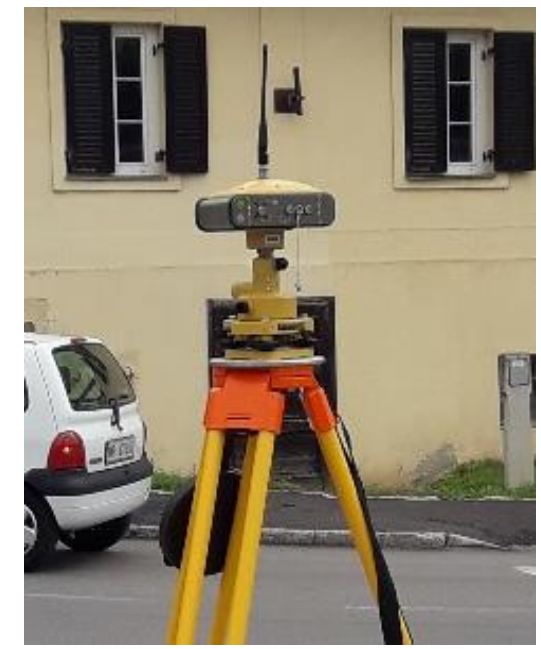

Fig. 7. Base station Topcon Hyper Pro.

\section{Results}

Dynamic oscillations were forced by two people with a total weight of $180 \mathrm{~kg}$, who were equally running or walking. The performance of the measurements in most scientific and engineering fields characterize the periodic signal of measured objects, where the periodicity is identified through spectral analysis. Spectral analysis, thus, presents a method of analysis using mathematical parameterization, where the chosen object is studied from the aspect of frequencies or other, indirectly connected quantities, such as energy, natural values, and so on.

The most represented and overall used technique in the spectral analysis area is the method of calculating with the help of Fourier transformation, which is applicable for continued, as well as direct, signals. The main limitation of the adequate use of Fourier transformation is that it demands a constant (equivalent) measuring interval [35].

Fourier transformation presents an extremely strong mathematical tool, which is used in numerous areas. The main reason for the overall use of this method is mostly that, by Fourier transformation, complicated operations of disposition and convolution are converted into simple multiplicative operation and, as such, Fourier transformation is an important tool in solving differential equations. In relevant scientific fields, this method is mostly used to determine the so-called frequency (oscillation) spectrum with the presence of a certain noise, where the data capture is, in principle, equal.

At present, it is possible to execute equal sampling with only advanced automatized measuring systems, such as accelerometers or strain gauges. On contrary to the latter, there is a problem with geodetic methods: there is commonly unequal data capture, where data are obtained over uneven or incorrect time intervals. Incorrect registration intervals and consequent sampling errors happen due to the natural technical flaws in the geodetic instruments. These errors are visually seen as so-called rough errors, which are the 
consequence of too-small frequencies, semi-automatization, noise, and mechanical measurement interruption.

Fourier transformation, whether of discrete or fast type, becomes practically useless when we want to consider data collected at unequal intervals in the analysis; which can be obtained from various geodetic measuring machines, such as RTS

As a solution to include and consider the unevenness of the sampling in data processing, the Lomb periodgram method was introduced by Nicholas R. Lomb and Jeffrey D. Scargle. This method can be used to allow for the spectral analysis of incorrectly (as well as correctly) sampled measurement data.

The Lomb (or Lomb-Scargle) periodogram is a mathematical method based on the principle of smallest squares for assessing the frequency spectra of random oscillations and the periodicity of even and uneven samplings. The procedure is based on matching sinusoidal functions of the shape [36, 37]:

$$
h(t)=a \cdot \cos \left(\frac{2 \pi}{T} \cdot(t-\tau)\right)+b \cdot \sin \left(\frac{2 \pi}{T} \cdot(t-\tau)\right) .
$$

The Lomb periodogram $P(t)$ describes a non-dimensional value, with which the spectral power is expressed. For time period $T$, it is defined as [36, 37]:

$$
\begin{aligned}
P(T)=\frac{1}{2 \sigma^{2}} \times\left\{\frac{\left[\sum_{j=1}^{W}\left(x_{j}-\bar{x}\right) \cdot \cos \frac{2 \pi\left(t_{i}-\tau\right)}{T}\right]^{2}}{\sum_{j=1}^{N} \cos ^{2} \frac{2 \pi\left(t_{i}-\tau\right)}{T}}\right. \\
\left.+\frac{\left[\sum_{j=1}^{W}\left(x_{j}-\bar{x}\right) \cdot \sin \frac{2 \pi\left(t_{i}-\tau\right)}{T}\right]^{2}}{\sum_{j=1}^{W} \sin ^{2} \frac{2 \pi\left(t_{i}-\tau\right)}{T}}\right\}
\end{aligned}
$$

where in the equation for the time lapse, the parameter $\tau$ is defined as:

$$
\begin{aligned}
& \tan \left(\frac{4 \pi \tau}{T}\right) \\
& =\frac{\sum_{j=1}^{W} \sin \left(\frac{4 \pi \tau_{i}}{T}\right)}{\sum_{j=1}^{W} \cos \left(\frac{4 \pi \tau_{i}}{T}\right)} .
\end{aligned}
$$

where:

$N$... the size of the data block;

$t_{i} \ldots$ time of the measured displacement $i$

$\bar{x} \ldots$ mean value of the displacement, calculated with the term $\bar{x}=\frac{\mathbb{1}}{N} \cdot \sum_{i=1}^{W} x_{i}$; and

$\sigma^{2} \ldots$ standard deviation, calculated with the expression $\sigma^{2}=\frac{1}{N-1} \cdot \sum_{i=1}^{N}\left(x_{i}-\bar{x}\right)^{2}$.

The obtained extreme positive values of the Lomb-Scargle periodogram, which appear in place of the frequencies which diminish the sum of squares of fitting the value to the timeline sinusoidal oscillation, give an approximation of the amplitude, as shown in figures $8,9,10$, and 11 and in Tables 3, 4, 5, and 6 .

This section may be divided by subheadings. It should provide a concise and precise description of the experimental results and their interpretations, as well as the experimental conclusions that can be drawn.

- Situation 1: Noise oscillations. The first rows of Figures 8, 9, 10, and 11 present the measured signal, the second rows show Lomb-Scargle periodogram (a spectral 
approximation for the area between $0-1 \mathrm{~Hz}$ of the basic signal), and the third presents $\mathrm{A}(\mathrm{f})$ for the whole Lomb-Scargle periodogram, where A(f) is calculated from Eq. 3.2.
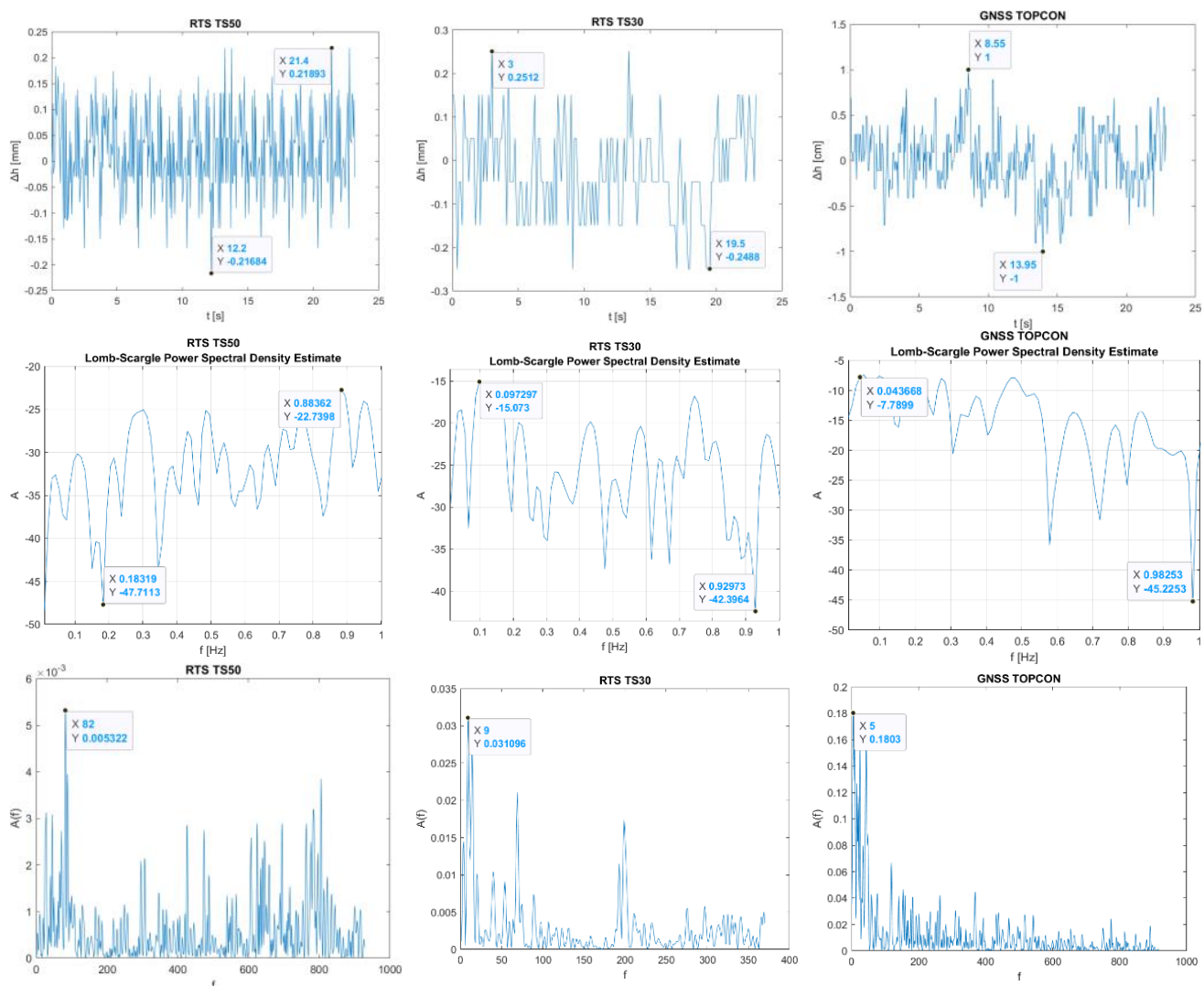

Fig. 8. Graphical displays of monitoring and spectral analysis in Situation 1.

Table 3. Results of the analysis of Situation 1 with geodetic methods.

\begin{tabular}{|c|c|c|c|c|c|c|c|}
\hline \multicolumn{7}{|c|}{ Noise oscillation } \\
\hline & \multicolumn{3}{|c|}{ Displacement $[\mathrm{mm}]$} & \multicolumn{3}{c|}{$\begin{array}{c}\text { Lomb-Scargle periodogram } \\
\text { (range 0-1 Hz) }\end{array}$} & \multirow{2}{*}{ A(f) } \\
\cline { 2 - 7 } & Min & Max & $\boldsymbol{\Delta}$ & Min & Max & $\Delta$ & \\
\hline TS50 & -0.21684 & $\mathbf{0 . 2 1 8 9 3}$ & $\mathbf{0 . 4 3 5 7 7}$ & $-\mathbf{- 4 7 . 7 1 1 3}$ & $\mathbf{- 2 2 . 7 3 9 8}$ & $\mathbf{2 4 . 9 7 1 5}$ & $\mathbf{0 . 0 0 5 3 2 2}$ \\
\hline TS30 & -0.2488 & $\mathbf{0 . 2 5 1 2}$ & $\mathbf{0 . 5 0 0 0}$ & $\mathbf{- 4 2 . 3 9 6 4}$ & $\mathbf{- 1 5 . 0 7 3 0}$ & $\mathbf{2 7 . 3 2 3 4}$ & $\mathbf{0 . 0 3 1 0 9 6}$ \\
\hline GNSS & -10.00 & $\mathbf{1 0 . 0 0}$ & $\mathbf{2 0 . 0 0}$ & $\mathbf{- 4 5 . 2 2 5 3}$ & $\mathbf{- 7 . 4 4 0 2}$ & $\mathbf{3 7 . 7 8 5 1}$ & $\mathbf{0 . 1 8 0 3 0 0 0}$ \\
\hline
\end{tabular}

- Situation 2: Walking over the bridge away from the measurement location. 

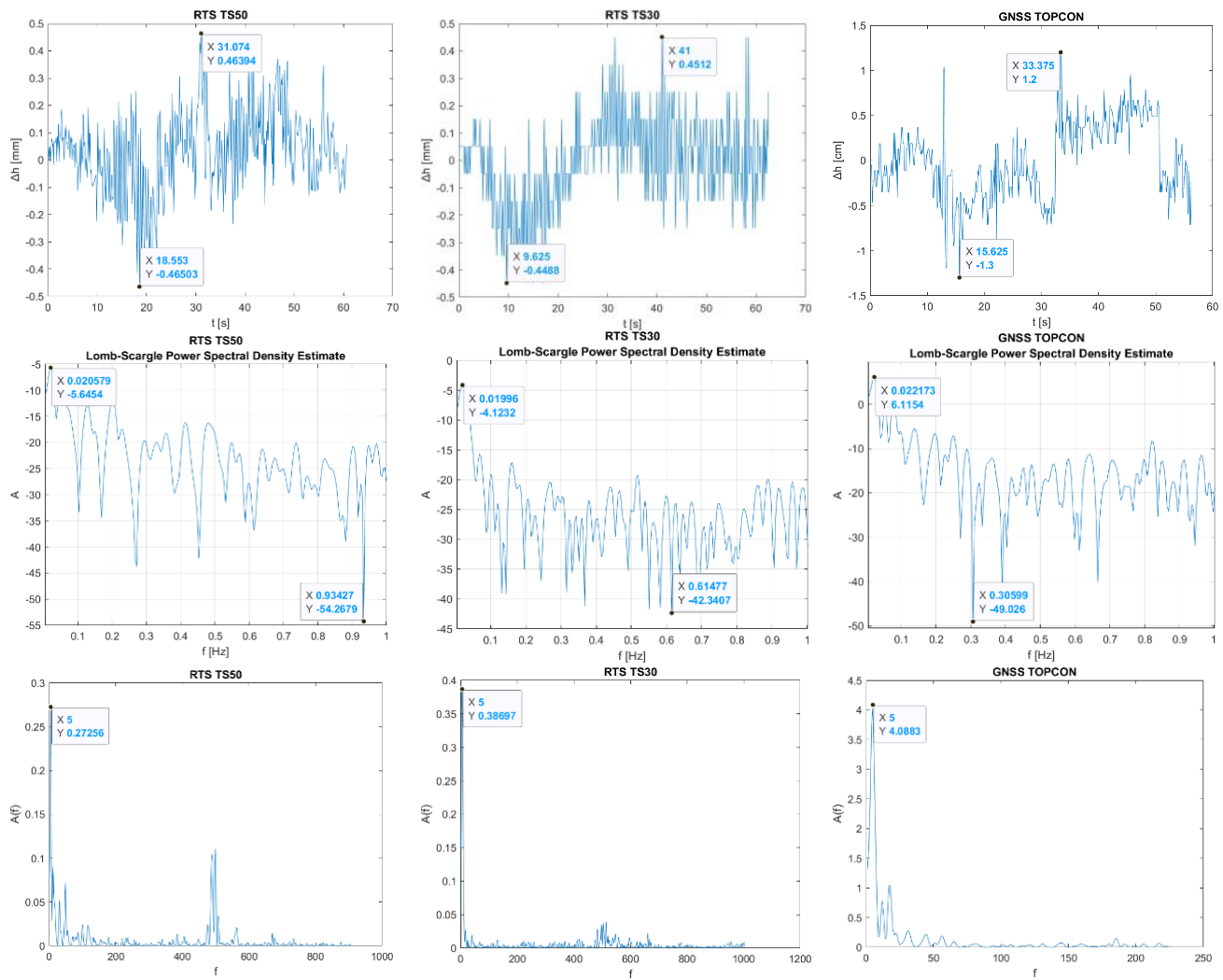

Fig. 9. Graphical displays of monitoring and spectral analysis in Situation 2.

Table 4. Results of the analysis of Situation 2 with geodetic methods.

\begin{tabular}{|c|c|c|c|c|c|c|c|}
\hline \multicolumn{8}{|c|}{ Walking over the bridge away from the measurement location. } \\
\hline & \multicolumn{3}{|c|}{ Displacement [mm] } & \multicolumn{3}{c|}{$\begin{array}{c}\text { Lomb-Scargle periodogram } \\
\text { (range 0-1 Hz) }\end{array}$} & \multirow{2}{*}{ A(f) } \\
\cline { 2 - 7 } & Min & Max & $\Delta$ & Min & Max & $\Delta$ & \\
\hline TS50 & -0.4650 & 0.4639 & 0.9289 & -54.2679 & -5.6454 & 48.6225 & 0.27256 \\
\hline TS30 & -0.4488 & 0.4512 & 0.9000 & -42.3407 & -4.1232 & 38.2175 & 0.38697 \\
\hline GNSS & -13.00 & 12.00 & 25.00 & -49.0260 & 6.1154 & 55.1414 & 4.0883 \\
\hline
\end{tabular}

- Situation 3: Running over the bridge away from the measurement location. 

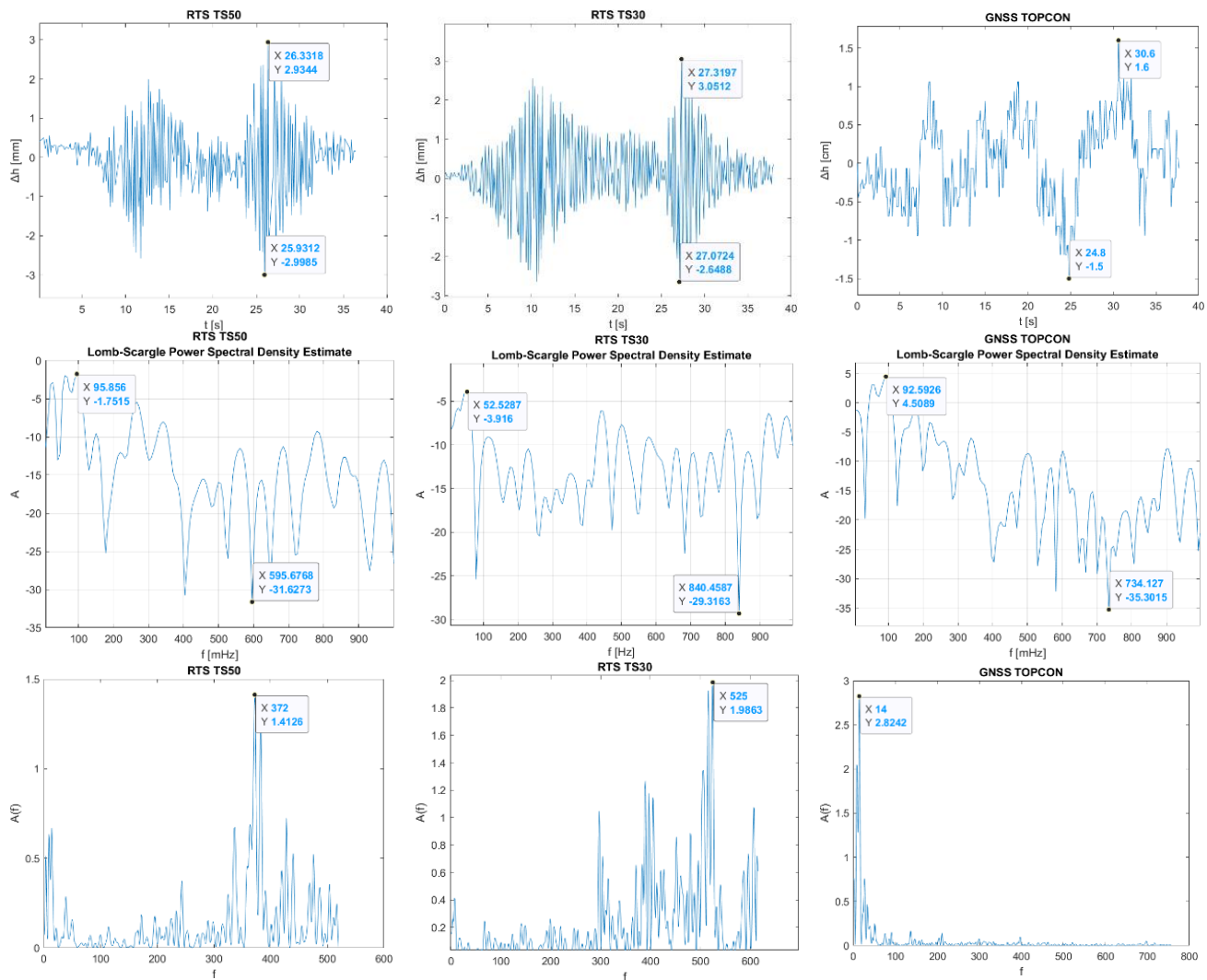

Fig. 10. Graphical displays of monitoring and spectral analysis in Situation 3.

Table 5. Results of the analysis of Situation 3 with geodetic methods.

\begin{tabular}{|c|c|c|c|c|c|c|c|}
\hline \multicolumn{9}{|c|}{ Running over the bridge away from the measurement location. } \\
\hline \multirow{2}{*}{} & \multicolumn{2}{|c|}{ Displacement [mm] } & \multicolumn{2}{c|}{$\begin{array}{c}\text { Lomb-Scargle periodogram } \\
\text { (range 0-1 Hz) }\end{array}$} & \multirow{2}{*}{ A(f) } \\
\cline { 2 - 7 } & Min & Max & $\boldsymbol{\Delta}$ & Min & Max & $\Delta$ & \\
\hline TS50 & -2.9985 & 2.9344 & 5.9329 & -31.6273 & -1.7515 & 29.8758 & 1.4126 \\
\hline TS30 & $-2,6488$ & 3.0512 & 5.7000 & -29.3163 & -3.916 & 25.4003 & 1.9863 \\
\hline GNSS & -19.00 & 8.00 & 27.00 & -35.3015 & 4.5089 & 39.8104 & 2.8242 \\
\hline
\end{tabular}

- Situation 4: Running over the bridge towards the measurement location. 

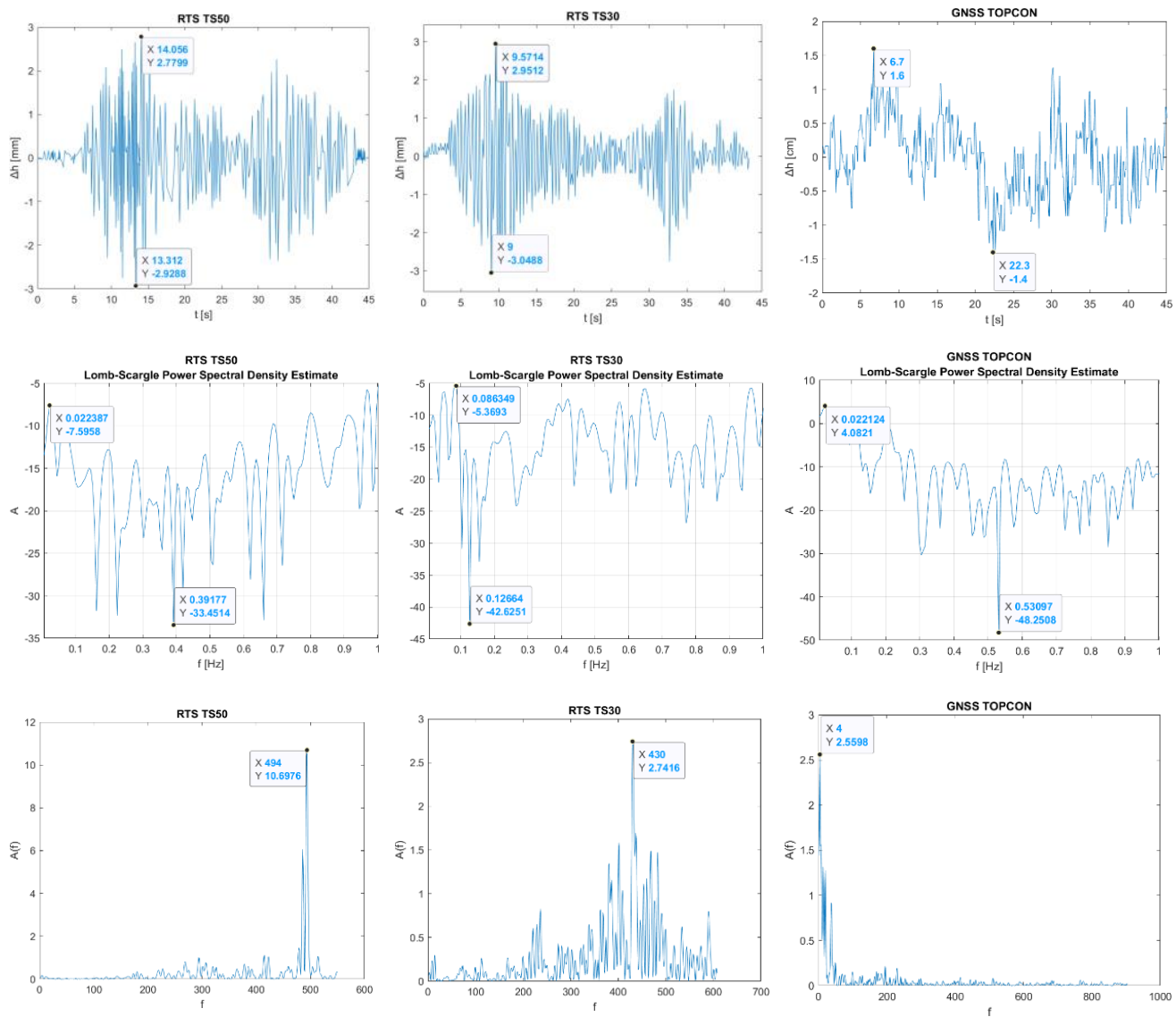

Fig. 11. Graphical displays of monitoring and spectral analysis in Situation 4.

Table 6. Results of the analysis of Situation 4 with geodetic methods.

\begin{tabular}{|c|c|c|c|c|c|c|c|}
\hline \multicolumn{8}{|c|}{ Running over the bridge towards the measurement location. } \\
\hline & \multicolumn{3}{|c|}{ Displacement [mm] } & \multicolumn{2}{c|}{$\begin{array}{c}\text { Lomb-Scargle periodogram } \\
\text { (range 0-1 Hz) }\end{array}$} & \multirow{2}{*}{ A(f) } \\
\cline { 2 - 7 } & Min & Max & $\boldsymbol{\Delta}$ & Min & Max & $\Delta$ & \\
\hline TS50 & -2.9288 & 2.7799 & 5.7087 & -33.4514 & -7.5958 & 25.8556 & 10.9676 \\
\hline TS30 & -3.0488 & 2.9512 & 6.0000 & -42.6251 & -5.3963 & 37.2288 & 2.7416 \\
\hline GNSS & -14.00 & 16.00 & 30.00 & -48.2508 & 4.0821 & 52.3329 & 2.5598 \\
\hline
\end{tabular}

The smallest obtained oscillation amplitude was $0.43 \mathrm{~mm}$. At such small displacement values, a GNSS system will not give good quality results. Worse results were obtained, due to the accuracy of the GNSS system in the vertical direction: the obtained oscillation amplitude (displacement) was smaller than the accuracy of the system in the vertical direction.

The advantage of the GNSS system vis-a-vis to the RTS system is evident at larger frequencies and oscillation amplitudes. Modern GNSS systems can record data up to 100 $\mathrm{Hz}$.

From Figures 8-11 and Tables 3-6, it can be seen that the GNSS system had a natural oscillation of $20 \mathrm{~mm}$ in the vertical direction. If this value is deducted from all obtained values of our experiment, comparable values would be obtained. However, in such 
demanding engineering works, where the damage of bridges are assessed, this is not an option.

\section{Discussion}

Based on presented and analyzed responses of the structure on certain burdening cases and the performed spectral analysis of individual signals, a practical value can finally be given. In almost all burdening cases, analysis of the results obtained by RTS TS50 showed a worse sampling frequency compared to the declared values, which suggests the possibility that measurements even up to $30 \mathrm{~Hz}$ were detected by using the GeoCOM/ZG program. The average sampling frequency during the whole experiment was $16 \mathrm{~Hz}$. From the results of the monitoring of dynamic response with non-contact geodetic methods, it can be seen that a high accuracy of measurement at lower oscillation frequencies was reached; however, accuracy decreased with increased oscillation frequencies. Based on this, it can be concluded that the accuracy in determining the dynamic response and oscillation frequency is dependent on the movement speed of the prism.

\section{Conclusion}

The monitoring of objects in modern building practice presents an extremely important area of the analysis of building structures, based on which a general insight into the structure's conduct may be obtained; however, this is susceptible to numerous effects during the examination period. Assessed or measured characteristics can now be exploited using numerous analyses and improvements and, based on the latter, potential negative consequences can be prevented. Following the rapid technological development of building procedures, which has been expressed in more and more statically demanding and bigger structures, methods for the analysis of structural response have been simultaneously developed, as well.

Correct experimental design and further analysis of measured signals of a footbridge structure, which was burdened over four different mutually independent events, comprised the main goal of this experiment. By assessing the potential co-ordination of the obtained results, the monitoring was performed with three different geodetic instruments, two robotic total stations (RTS Leica TS50 and RTS Leica TS30) and a GNSS receiver (Topcon Hiper Pro), along two different physical instruments, the Instantel Minimate Plus and Rotech Micro Mon accelerometers. According to the theoretical principles of absolute and relative methods, it was discerned, before the execution itself, that a comparison between the geodetic and physical methods would be hardly doable. Due to this, the analysis was performed separately for each type of the method.

Monitoring of the Studenci Footbridge was performed, simultaneously and uninterruptedly, with all of the geodetic measuring instrumentation, as well as physical methods. Due to the simultaneous initiation, it turned out that the processing and synchronization of the results was somewhat easier, due to the clear amplitude extremes and physicality of the signals. In most cases, in the application of the GNSS receiver, it turned out that millimetre-scale oscillation amplitudes presented values too small, due to the technical characteristics of chosen measuring equipment. Additionally, it turned out that, in the case of total robotic stations with direct measuring, relative vertical displacements were obtained, as opposed to absolute values, as in the case of the GNSS system. It was determined that, if the previously measured natural oscillation was deducted from the obtained results of the GNSS receiver, relatively good approximations, with reference to the measurements of the RTS, could be obtained. Special attention was paid to 
the analysis of the response, as well as in the case of testing, in order to assess the accuracy of the measurements, as affected by the sampling frequency. It was concluded that, in mutual comparison of the obtained values at $6 \mathrm{~Hz}$ or $26 \mathrm{~Hz}$, relatively exceptionally small changes were observed, from which it is concluded that frequency has a certain effect on the accuracy of the measurements, but this was, in the considered case and for the needs of general practice, evaluated as negligibly small.

As has already been mentioned, a noticeable difference would be possible to note in the comparison of GNSS measurements, according to the results obtained with RTS. The deviations, even after pre-determined natural values were deducted, were still noticeable, from which it was concluded that the application of GNSS and indirectly connected GPS systems are much more meaningful for monitoring larger displacements or amplitudes over longer time periods.

The application of spectral analysis procedures and methods, such as Fourier transformation and the Lomb-Scargle algorithm, have turned out to be strong mathematical tools for the analysis and interpretation of the frequency components of structural oscillations.

A small flaw was assessed, in the case of sampling with the application of the robotic total station RTS Leica TS50, where, due to a short interruption of measurements or unevenness, a deviation occurred. In the case of Studenci Footbridge monitoring, all results of the responses of the footbridge following different burdening events with the mentioned measurement equipment, were mutually comparable, and an almost perfect synchronization was enabled in the case of geodetic methods.

Monitoring of the object using our experimental set-up helped us to achieve representative and correct results (from an engineering perspective); thus, it is definitely reasonable to execute monitoring with the application of geodetic, as well as physical, methods, as this is likely the only way to obtain a fuller insight into the conduct and response of the structure over its exploitation period.

\section{References}

1. W. Lienhard, M. Ehrhart, M. Grick, Proceedings of 3rd Joint International Symposium on Deformation Monitoring (JISDM) (Vienna, Austria, 2016)

2. W. Lienhard, M. Ehrhart, Proceeings of International Workshop of Structural Health Monitoring (IWSHM) (Stanford, USA, 2015)

3. M. Celebi, A. Sanli, Earthq Spectra 18(1), 47-61 (2002)

4. Q. Chen, D.F. Huang, X.L. Ding, Y.L. Xu, J.M. Ko, Proceedings of Health monitoring and management of civil infrastructure systems (Bellenham (WA), SPIE, 2001)

5. G.W. Roberts, X. Meng, A.H. Dodson, 10th FIG International Symposium on Deformation Measurement (Orange, California, 2001)

6. C. Ogaja, J. Wang, C. Rizos, Journal of Surveying Engineering 129(3), 99-104 (2003)

7. X. Meng, A.H. Dodson, G.W. Roberts, Engineering Structures 29, 3178-3184 (2007)

8. A. Marendič, Z. Kapović, R. Paar, Geodetski list 3, 175-190 (2013)

9. E.J. Cross, K.Y Koo, J.M.W. Brownjohn, K. Worden, Mechanical Systems and Signal Processing 35(1-2), 16-34 (2013)

10. K.Y. Koo, J.M.W. Brownjohn, Structural Control and Health Monitoring 20, 609-625 (2013)

11. S. Psimoulis, S. Stiros, Journal of Bridge Engineering 18, 182-185 (2013) 
12. A. Marendić, R. Paar, I. Grgac, D. Damjanović, Proceedings of 3rd Joint International Symposium on Deformation Monitoring (JISDM) (Vienna, Austria, 2016)

13. A. Marendić, R. Paar, I. Duvnjak, A. Buterin, 6th International Conferece on Engineering Surveying (FIG, Prague, Czech Republic, 2014)

14. P. Psimoulius, S. Stiros, Engineering Structures 29, 3312-3324 (2007)

15. W. Stempfhuber, Proceedings of 3rd International Symposium on Mobile mapping Technology (Cairo, 2000)

16. R.S. Radovanović, W.F. Teskey, Proceedings of 10th symposium of deformation measurements (Orange, California, 2001)

17. A. Kopačik, P. Kyronović, V. Kadlecikova, Proceedings of FIG working week (Cairo, 2005)

18. I. Ceryova, P. Kubanka, A. Kopačik, P. Kyrinovič, Dynamic test of Robot stations. International Congress of deformations measurement (FIG, Washington. D.C., USA, 2002)

19. S.C. Chua, Testing of Robotic Total Stations for Dynamic Tracking, Doctoral Thesis (University of Southern Queensland, Australia, 2004)

20. V. Gikas, S. Daskalakis, 13th FIG congress shaping the change (Munich, 2006)

21. E. Cosser, G.W. Roberts, X. Meng, A.H. Dodson, 11th FIG symposium of deformation monitoring (Santorini, Greece, 2003)

22. V. Lekidis, M. Tsakiri, K. Makra, C. Karakostas, N. Klimis, I. Sous, Engineering Geology 179, 7-17 (2005)

23. B. Schmitz, C. Holst, T. Medic, D.D. Lichti, H. Kuhlmann, Sensors 19(6), 1466 (2019)

24. Z. Yi, C. Kuang, Y. Wang, W. Yu, C. Cai, W. Dai, Sensors 18(12) (2018)

25. X. Yang, K. Steward, L. Tang, Z. Xie, Q. Li, Sensors 18(11), 3741 (2018)

26. G. Novakovic, A. Marendić, I. Grgac, R. Paar, R. Ilijaš, Geodetski list 69(4), 279-304 (2015)

27. A. Marendić, R. Paar, D. Damjanović, Građevinar 69(4), 281-294 (2017)

28. F. Poyraz, Technical Gazette 23(1), 107-114 (2016)

29. I. Grgić, M. Repanić, I. Malović, Technical Gazette 24(1), 301-308 (2017)

30. A. Guntel, H. Karabork, L. Karasaka, Technical Gazette 25(1), 66-74 (2018)

31. V. Ilci, I. Ozulu, E. Arslan, R. Alkan, Technical Gazette 25(6), 1642-1649 (2018)

32. P. Psimoulius, S. Pytharouli, D. Karambalis, S. Stiros, Journal of Sound Vibration 318(3), 606-623 (2008)

33. P. Psimoulius, S. Stiros, 4th International Conference on Engineering Surveying INGEO (Bratislava, Slovakia, 2008)

34. C. Heinemeyer, C. Butz, A. Keil, M. Schlaich, and others, JRC Scientific and technical Reports JRC 53442 (2009)

35. A. Marendić, Primjena geodetskih mjernih sustava u nadgledanju građevina $s$ naglaskom na praćenje dinamičkih pomaka, Doctoral Thesis (University of Zagreb, Croatia, 2011)

36. W.H. Press, S.A. Teukosky, W.T. Vellerling, B.P. Flannery, The Mathematical Gazette 73(464), 167-170 (1988)

37. N.R. Lomb, Astrophys Space Sci. 39, 447-462 (1976) 